\title{
Bi Lateral Vocal Cord Paralysis Post Thyroidectomy Causing Total Airway Obstruction: Case Report
}

\author{
Surbhi D. Mundada*, Kundan S. Gosavi and Naveed J. Khan
}

Department of Anaesthesia and Critical Care, Grant Medical College and Sir. J. J. Groups of Hospitals, Mumbai8, India

\begin{abstract}
Thyroid surgery in recent years is generally considered quite safe. Better preoperative preparation and proper surgical techniques of thyroidectomy have minimized complications to less than $2-3 \%$. Bilateral recurrent nerve palsy is the rarest of all complications, with a reported incidence varying between $0.4 \%$ to $14 \%$, but can be potentially fatal if missed $[1,2]$.

We report a case in which the patient experienced life threatening stridor and severe dyspnoea requiring re-intubation few minutes after extubation.
\end{abstract}

Keywords: Vocal cord palsy, total thyroidectomy, airway obstruction.

\section{CASE REPORT}

A 29 year old female patient, weighing $48 \mathrm{~kg}$, presented with a thyroid swelling of about $3 \times 3 \mathrm{~cm}$ in size, that has existed for six months. No retrosternal extension or pressure symptoms were observed. Following her previous diagnosis of thyroid hyperfunction she has been treated with Carbimazole and Propanolol for six months. Presently she was asymptomatic and her thyroid function tests were normal. Preoperative indirect laryngoscopy showed normal cord movements. X-ray of the neck did not show any deviation or tracheal compression. The preoperative histopathology report was suggestive of malignancy.

On the day of surgery her pulse rate was 78 beats per minute, rhythmic and her blood pressure was $130 / 86 \mathrm{mmHg}$. After securing an intravenous (i.v) line, $1 \mathrm{mg}$ of Midazolam and $100 \mathrm{mcg}$ of Fentanyl were administered intravenously. Anesthesia was induced with $100 \mathrm{mg}$ of Propofol and intubation was facilitated by administereing $75 \mathrm{mg}$ suxamethonium. Anesthesia was maintained with $\mathrm{N}_{2} \mathrm{O}, \mathrm{O}_{2}(60: 40)$ and $2 \%$ sevoflurane. Vecuronium was used as muscle relaxant and the intraoperative anesthetic course was uneventful.

The surgery was performed without complications and lasted for about 3 hours. Before closure, a negative suction drain was inserted into the wound and pressure dressing was applied after surgery.

At the end of surgery, neuromuscular blockade was reversed with $2.5 \mathrm{mg}$ Neostigmine in combination with $0.2 \mathrm{mg}$ Glycopyrolate. Once a Train of four (TOF-) ratio of 0.9 was measured, and the patient while intubated showed a normal tidal volume and respiratory rate. After extubation, the patient was obeying commands but was unable to speak

*Address correspondence to this author at the Department of Anaesthesia and Critical Care, Sir. J. J. Group of hospitals Address: 51- Rohini bldg, NOFRA Colaba (Navy nagar) Mumbai- 400005; Tel: 9869322731 ; E-mail: sur_mahe@hotmail.com and developed a stridor with severe dyspnoea after a few minutes. The oxygen saturation dropped to $60 \%$ and the patient received $100 \%$ oxygen via face mask but the treatment neither improved her dyspnoea nor her saturation. Chest indrawing while breathing was visible and $50 \mathrm{mg}$ suxamethonium were injected for reintubation. Tracheal compression by an hematoma was part of our differential diagnosis but was considered unlikely based on the clinical exam : after removal of the pressure dressing a clean suture lineno visible hematoma and no oozing from the wound were observed. IV administration of $8 \mathrm{mg}$ dexamethasone and $10 \mathrm{ml} \mathrm{Calcium}$ gluconate $(10 \%)$ also took place prior to intensive care unit admission.

After 24hrs, another extubation trial failed such that the patient was tracheostomized which relieved the patient's respiratory distress.

She was taken to the operating theatre the next day and nasal fiberoptic laryngoscopy was performed under midazolam sedation. The exam showed both vocal cords in an adducted position. There was no laryngeal edema and thus the diagnosis of bi-lateral recurrent nerve palsy was made.

The vocal cord paralysis was transient: tracheostomy tube could be removed and the ability to speak normally recovered during six months following thyroid surgery.

\section{DISCUSSION}

Thyroid surgery is a common surgical procedure in India. Improvements in techniques over the years have reduced the mortality rate near to zero. Morbidity, however, always remains a concern. The incidence of injuries to the recurrent laryngeal nerve is about $1-14 \%[2,3]$. Bilateral Recurrent Laryngeal Nerve (RLN) injury is rare and could lead to severe, life threatening complications. In this condition, both vocal cords remain in a median or paramedian position. As a result, the patient can develop inspiratory stridor, dyspnoea, tachypnoea, and nasal flaring $[4,5]$. Sole dysphonia without 
dyspnoea could also be a possible observation [10]. In institutions where direct laryngoscopy is not performed as a standard procedure to visualize vocal cords after surgery, the diagnosis of bilateral paralysis can be missed if there is an inability to visualize vocal cords due to a missing patient cooperation, for instance. In this case, valuable time is lost before making a definitive diagnosis.

Risk factors highly associated with an increased incidence of post operative bilateral RLN palsy include:

\section{Incomplete Dissection or Exposure of RLN}

By adopting the principle of routine exposure of RLN, a very low nerve injury rate has been reported in the literature, even after total thyroidectomy performed for thyroid cancer $[6,7]$.

\section{Total Throidectomy and Thyroid Cancer}

It has been reported that prevalence of RLN injury increases with the size of the pathologic gland, thyroid cancer and extent of thyroid resection $[3,8,9]$.

\section{Redo Surgery and Ligature of the Inferior Thyroid Artery [10] also Increase the Chance of RLN Injury}

Our patient presented with thyroid cancer for total thyroidectomy which put her at increased risk for bilateral palsy. The thyroid swelling was moderate, had not been present for a very long time and surgeons confirmed that no softening of tracheal rings were detectable during surgery. Therefore, we ruled out tracheomalacia as a potential cause for the postoperative dyspnoea.

Laryngospasm after extubation, possilby due to hypocalcemia, could be another possible explanation for the developed stridor. However, after the administration of succinylcholine initially and Calcium gluconate as a bolus consequently, blood samples were drawn and normal levels for total serum calcium and ionized calcium were revealed, which makes this an unlikely mechanism to have caused the stridor. Laryngeal edema could have been another cause for the events. We therefore transferred the patients to the ICU for another extubation attempt after $24 \mathrm{hrs}$ and treated her with $4 \mathrm{mg}$ Dexamethasone 8 hourly. Nontheless, these attempts did not influence the patient's inability to maintain a patent airway, and eventually an examination with fiberoptic laryngoscopy in the OR verified the diagnosis of bilateral vocal cord palsy.

Possible causes could include accidental compression or damage to the recurrent laryngeal nerve during surgery, leading to nerve neuropraxia or accidental dissection of the nerve, a rare complication during resection of the thyroid gland. A better understanding and estimation of the factors putting the patient at high risk for recurrent nerve palsy and a better assessment of the vocal cords postoperatively would allow for a faster detection and improved handling of the observed postoperative complications in this case report.

Transient paralysis can often be seen to recover after 6 to 8 months. Hence, a follow up with direct or indirect laryngoscopy for at least 6 months might improve the outcome of these cases. In case of permanent paralysis, surgical intervention remains the only option for treatment. Endoscopic ventriculocordectomy, or arytenoidectomy are the surgical procedures of choice for such patients. The use of electrophysiological monitoring of RLN during thyroid surgery has been mentioned in the literature in high risk cases to avoid inuring the nerve $[1,9]$

To conclude, bi-lateral vocal cord palsy is a rare but possible complication in the course of recurrent thyroid surgery, total thyroidectomy, or thyroid carcinoma. Knowledge about the risk factors contributing to this complication, might increase awareness for such an event, thus allowing early intervention to avert adverse or fatal outcomes.

\section{REFERENCES}

[1] Robertson ML, Steward DL, Gluckman JL, Welge J. Continuous laryngeal nerve integrity monitoring during thyroidectomy: Does it reduce risk of injury? Otolaryngol Head Neck Surg 2004; 131(5): 596-600.

[2] Myssiorek D. Recurrent Laryngeal nerve paralysis: anatomy and etiology. Otolaryngol Clin N Am 2004; 37(1): 25-44

[3] Trésallet C, Chigot JP, Menegaux F. How to prevent recurrent laryngeal nerve palsy during thyroid surgery. Ann Chir 2006; 131(2): 149-53.

[4] Sinagra DL, Montesinos MR, Tacchi VA, et al. Voice changes after thyroidectomy without recurrent laryngeal nerve injury. J Am Coll Surg 2004; 199(4): 556-60.

[5] Schulte KM, Roher HD. Complications in the surgery of benign thyroid disease. Acta Chir Austriaca 2001; 33(4): 164-72.

[6] Wheeler MH. Thyroid surgery and recurrent laryngeal nerve. Br J Surg 1999; 86(3): 291-2.

[7] Hermann M, Alk G, Roka R, et al. Laryngeal recurrent nerve injury in surgery for benign thyroid disease: effect of nerve dissection and impact of individual surgeon in more than 27,000 nerves at risk. Ann Surg 2002; 235(2): 261-8.

[8] Hermann M, Hellebart C, Freissmuth M. Neuromonitoring in thyroid surgery: Prospective evaluation of intraoperative electrophysiological responses for the prediction of recurrent laryngeal nerve injury. Ann Surg 2004; 240(1): 9-17.

[9] Chou FF, Su CY, Jeng SF, et al. Neurorrhaphy of the recurrent laryngeal nerve. J Am Coll Surg 2003; 197(1): 52-7.

[10] Zakaria HM, Al Awad NA, Al Kreedes AS, et al. Recurrent Laryngeal Nerve Injury in Thyroid Surgery. Oman Med J 2011; 26 (1): 34-8. 